\title{
Morbimortalidade do Idoso Internado na Unidade de Terapia Intensiva de Hospital Universitário de Fortaleza*
}

\author{
Morbidity and Mortality of Elderly Patients Admitted to an \\ Intensive Care Unit of a University Hospital in Fortaleza
}

\author{
Carlos Augusto Ramos Feijó1, lara Serra Azul Machado Bezerra1, \\ Arnaldo Aires Peixoto Júnior ${ }^{2}$, Francisco Albano de Meneses $^{3}$
}

\section{RESUMO}

JUSTIFICATIVA E OBJETIVOS: Identificar a gravidade dos pacientes idosos atendidos na Unidade de Terapia Intensiva (UTI) de um hospital universitário, relacionando com a mortalidade durante a internação.

MÉTODO: Foi realizado um estudo retrospectivo, com análise de 130 pacientes admitidos na UTI, no período de março de 2004 a julho de 2005.

RESULTADOS: Dos 130 pacientes, houve predomínio do sexo feminino, com média de idade de 72,2 $\pm 7,3$ anos. Houve maior prevalência de pacientes na faixa entre 65 e 74 anos. Mais de $80 \%$ eram provenientes do próprio hospital universitário. De acordo com os sistemas acometidos, as principais disfunções foram cardiovasculares e respiratórias. A sepse foi implicada como causa em $23,8 \%$ dos pacientes. O tempo médio de permanência na UTI foi de $8,2 \pm 7,6$ dias. A média

1. Médico Residente em Medicina Intensiva do Hospital Universitário Walter Cantídio (HUWC) da Universidade Federal do Ceará (UFC);

2. Médico Assistente da Unidade de Terapia Intensiva (UTI) do HUWC

- UFC; Especialista em Medicina Intensiva - AMIB; Especialista em

Nutrição Parenteral e Enteral - SBNPE; Especialista em Geriatria

- SBGG; Mestrando em Farmacologia - UFC.

3. Chefe da UTI do HUWC; Coordenador da Residência Médica em Medicina Intensiva; Especialista em Medicina Intensiva - AMIB; Especialista em Nutrição Parenteral e Enteral - SBNPE; Mestre em Farmacologia - UFC.

*Recebido da Unidade de Terapia Intensiva do Hospital Universitário Walter Cantídio (HUWC) - UFC, Fortaleza, CE

Apresentado em 19 de abril de 2006

Aceito para publicação em 06 de setembro de 2006

Endereço para correspondência:

Dr. Carlos Augusto Ramos Feijó

Rua Álvaro Fernandes, 741 - Montese

60420-570 Fortaleza, CE

Fone: (85) 3491-9309

E-mail: cfeijo2001@yahoo.com.br

(C)Associação de Medicina Intensiva Brasileira, 2006 de índice APACHE II foi $18,2 \pm 7,2$. Menores valores de APACHE II, tempo de internação e mortalidade foram observados entre os pacientes com acometimento primariamente cardiovascular. Do total, $66,1 \%$ foram transferidos da UTI, 6,2\% evoluíram para o óbito com menos de $48 \mathrm{~h}$, e $27,7 \%$ após as primeiras $48 \mathrm{~h}$. A razão de mortalidade padronizada foi de 0,988 .

CONCLUSÕES: A faixa etária não determinou diferença significativa entre os valores de APACHE II, não esteve associada a maior mortalidade, nem com maior tempo de permanência na UTI. Os pacientes com disfunção cardiovascular apresentaram menores valores de APACHE II, tempo de internação e mortalidade na UTI.

Unitermos: idoso, prognóstico, UTI.

\section{SUMMARY}

BACKGROUND AND OBJECTIVES: To identify the severity of elderly patients admitted to the intensive care unit (ICU) in a university hospital, relating it to the in-ICU mortality.

METHODS: Retrospective study, with analysis of 130 patients admitted to ICU from March 2004 to July 2005.

RESULTS: Of the 130 patients, there was a predominance of women, and mean $72.2 \pm 7.3$ years. There were more patients between 65 and 74 years old. More than $80 \%$ of the patients had come from the university hospital itself. The main dysfunctions were from the cardiocirculatory and respiratory systems. Sepsis caused $23.8 \%$ of the admissions. Length of stay in ICU was $8.2 \pm 7.6$ days. The mean of APACHE II was 18.2 \pm 7.2. Lesser values of APACHE II, length of stay and mortality were observed in patients with cardiocirculatory dysfunction. The in-ICU mortality was $33.9 \%$, $6.2 \%$ before 48 hours. The standardized mortality ratio (SMR) was 0.988 . 
CONCLUSIONS: The age groups did not determine difference between values of APACHE II. They were related neither to higher mortality rate, nor to higher ICU length of stay. Patients with cardiocirculatory dysfunctions had lesser values of APACHE II, ICU length of stay and in-ICU mortality.

Key Words: elderly, ICU, prognosis.

\section{INTRODUÇÃO}

A população de idosos vem crescendo nos últimos anos. No Brasil, a proporção de pessoas com 60 anos ou mais aumentou de 6,7\% em 1990 para 8,1\% em 2000; e as projeções do Instituto Brasileiro de Geografia e Estatística sugerem que essa população chegue a 64 milhões de pessoas em 2050, o que corresponderia a $24,6 \%$ do total de habitantes ${ }^{1}$. Essa tendência mundial vem alertando para a necessidade de melhorar os respectivos recursos em saúde, principalmente no que tange às medidas consideradas de alto custo, uma vez que os idosos são os maiores consumidores ${ }^{2}$.

A idade média dos pacientes de UTI tem aumentado nos últimos anos e aumentará ainda mais com o envelhecimento da população geral². Vários estudos demonstraram a relevância dos cuidados da Medicina Intensiva dispensados a pacientes geriátricos. Em países ocidentais, a percentagem de indivíduos acima de 65 anos representa $18 \%$ da população geral, respondendo por $45,5 \%$ das admissões hospitalares. Em relação a diárias de Unidade de Terapia Intensiva (UTI), cerca de $60 \%$ são consumidas por indivíduos acima de 65 anos de idade. Em pacientes acima de 75 anos, os custos por diária de UTI chegam a ser sete vezes superior, quando comparados com os de pacientes com idade inferior a 65 anos $^{3}$.

Este cenário justifica a preocupação não apenas com o amparo logístico ao idoso grave; mas também com o gerenciamento econômico e a real efetividade do suporte intensivo nesta população. Buscando melhorar o atendimento ao idoso crítico, objetivou-se, através deste estudo, identificar a gravidade dos pacientes geriátricos atendidos em nossa UTI, procurando conhecer fatores prognósticos associados a sua mortalidade durante a internação.

\section{MÉTODO}

O presente estudo foi realizado na UTI do Hospital Universitário Walter Cantídio (HUWC) da Universidade Federal do Ceará. A unidade possui seis leitos destinados a pacientes adultos e tem atendimento clínico-cirúrgico.
Com caráter descritivo e retrospectivo, analisaram-se 130 pacientes internados, de forma consecutiva, durante o período de 01 de março de 2004 a 31 de julho de 2005. Foram colhidas, no momento da admissão, as variáveis demográficas (sexo e idade), assim como procedência (se do próprio HUWC ou de outras unidades de saúde). Quanto aos fatores possivelmente associados à gravidade, foram identificados, de acordo com Knaus e col. ${ }^{4}$, a disfunção aguda que motivou a internação na unidade, - APACHE II calculado nas primeiras 24 horas da internação, a mortalidade prevista por este escore e a razão de mortalidade padronizada (SMR). Quanto ao sistema orgânico acometido, foram distribuídos em: (1) neurológico (acidente vascular encefálico, hemorragia subaracnóidea, hematoma subdural, trauma cranioencefálico, síndrome de Guillain-Barré); (2) cardiovascular (insuficiência cardíaca descompensada, síndrome coronariana aguda, disritmias, pós-parada cardiorrespiratória, emergências hipertensivas, embolia pulmonar); (3) respiratório (insuficiência respiratória aguda); (4) gastrintestinal (hemorragia digestiva, pancreatite); e (5) renal/metabólico (insuficiência renal aguda, cetoacidose diabética, intoxicação exógena). Deliberadamente, buscou-se individualizar a associação com eventos sépticos 5 . Procurou-se identificar uma correlação entre os fatores descritos e a mortalidade geral precoce ( $<48$ horas) ou tardia ( $>48$ horas), assim como o tempo de permanência na UTI.

O programa de computação SPSS for Windows (versão 11.0) foi usado para realizar a análise estatística. Média e desvio-padrão foram calculados para variáveis contínuas com distribuição normal; enquanto que a distribuição das proporções foi calculada para variáveis discretas. O teste do Qui-quadrado foi utilizado para avaliar variáveis qualitativas e categoriais, enquanto a Análise de Variância (ANOVA) foi usada para comparar médias de variáveis contínuas. O nível de significância adotado foi de $5 \%(\alpha=0,05)$. Nos testes estatísticos utilizados, foi calculado o valor de $\mathrm{p}$ e do risco relativo com intervalo de confiança de $95 \%$. O presente trabalho recebeu a aprovação da Comissão de Ética do Hospital Universitário Walter Cantídio.

\section{RESULTADOS}

Do total de pacientes estudados, houve predomínio do sexo feminino. A idade variou entre 60 e 93 anos, com média de 72,2 $\pm 7,3$ anos. Com relação à faixa etária, encontrou-se um maior número de pacientes entre 65 e 74 anos, enquanto apenas 3,8\% da população estudada tinham 85 anos ou mais. A maioria dos pacientes procedeu das en- 
fermarias do próprio HUWC $(80,8 \%)$. O tempo médio de permanência na UTI foi de 8,2 $\pm 7,6$ dias (Tabela 1).

Tabela 1 - Dados Demográficos

\begin{tabular}{lc}
\hline Idade (anos) $^{*}$ & $72,2 \pm 7,3$ \\
Grupos etários $^{+}$ & \\
$60-64$ anos & $24(18,5 \%)$ \\
$65-74$ anos & $51(39,2 \%)$ \\
$75-84$ anos & $50(38,5 \%)$ \\
$>84$ anos & $5(3,8 \%)$ \\
Sexo feminino & $69(53 \%)$ \\
Procedência & \\
HUWC & $105(80,8 \%)$ \\
Outros hospitais & $25(19,2 \%)$ \\
Tempo de permanência na UTI (dias) & $8,2 \pm 7,6$ \\
\hline
\end{tabular}

* Média \pm DP

+Valor absoluto (percentagem)

As disfunções cardiovasculares e respiratórias mais freqüentemente justificaram o encaminhamento dos pacientes para a UTI. Ressalte-se que a sepse foi identificada em quase um quarto dos pacientes analisados (Tabela 2). Registrou-se menor tempo de permanência e mortalidade geral na UTI entre os pacientes com disfunção cardiológica (Figuras 1 e 2).

Tabela 2 - Indicadores de Gravidade

\begin{tabular}{lc}
\hline APACHE II* & $18,2 \pm 7,2$ \\
Disfunções que motivaram a internação & \\
Cardiológica & $29,2 \%$ \\
Respiratória & $21,5 \%$ \\
Neurológica & $17,7 \%$ \\
Renal / Metabólica & $4,6 \%$ \\
Gastrintestinal & $3,1 \%$ \\
Sepse $^{+}$ & $23,8 \%$ \\
\hline
\end{tabular}

* Média \pm DP

+ Percentagem

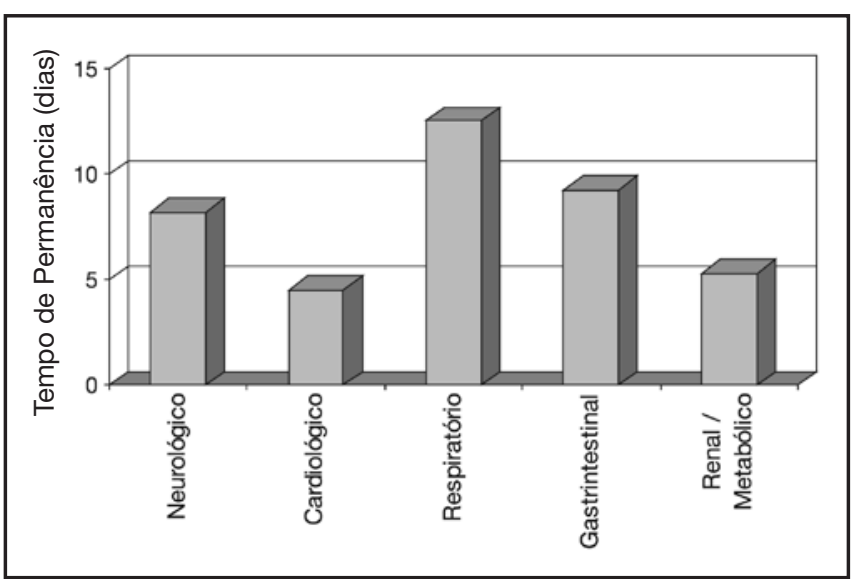

Figura 1 - Relação entre Disfunção e Permanência na UTI. $\mathrm{p}<0,05$

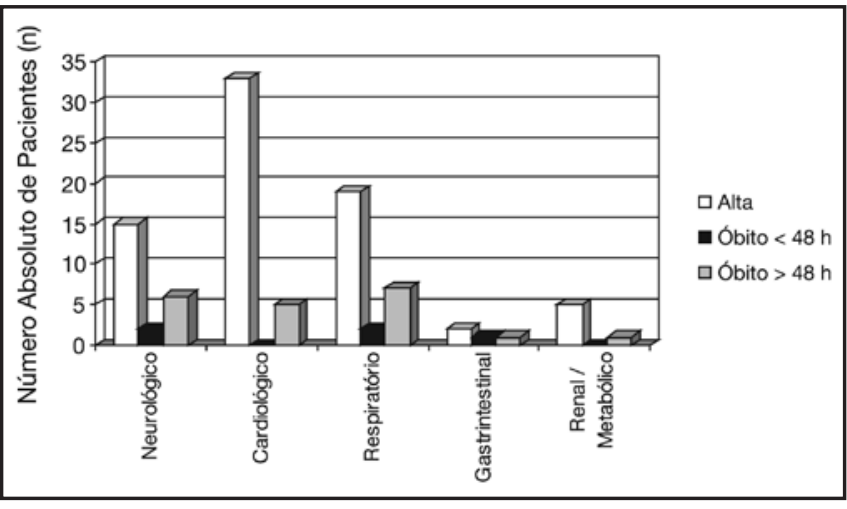

Figura 2 - Relação entre Disfunção e Óbito na UTI. $\mathrm{p}<0,05$

O índice APACHE II médio encontrado foi 18,2 $\pm 7,2$ e a mortalidade prevista, para a população estudada, de 34,1\% (Tabelas 2 e 3). Não houve diferença significativa entre o valor do APACHE II nos diferentes grupos etários. Os menores valores foram identificados entre os pacientes com disfunção cardiológica à admissão (Figura 3).

Tabela 3 - Mortalidade e Tempo de Permanência na UTI

\begin{tabular}{|c|c|}
\hline Mortalidade prevista* $^{*}$ & $34,1 \%$ \\
\hline Mortalidade real $^{\star}$ & $33,8 \%$ \\
\hline Óbito precoce $(<48 \text { horas })^{\S}$ & $8(6,2 \%)$ \\
\hline Óbito tardio (> 48 horas) $)^{\S}$ & $36(27,7 \%)$ \\
\hline Razão de mortalidade padronizada ${ }^{+}$ & 0,988 \\
\hline Alta da UT|§ & $86(66,2 \%)$ \\
\hline
\end{tabular}

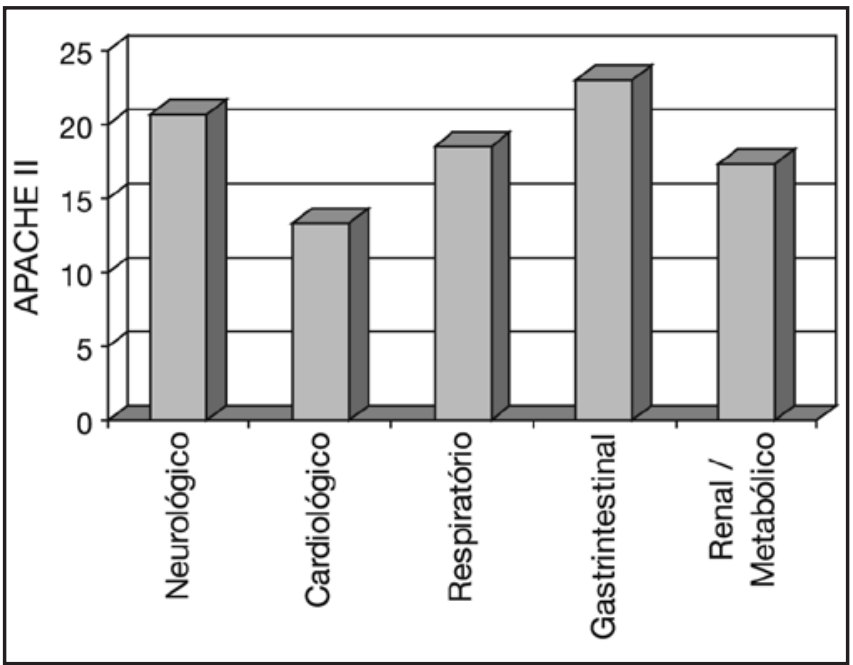

Figura 3 - Relação entre Disfunção e o Índice APACHE II. $p<0,05$ 
A idade não se associou significativamente com maior mortalidade nem com maior tempo de permanência na UTI (Figuras 4 e 5).

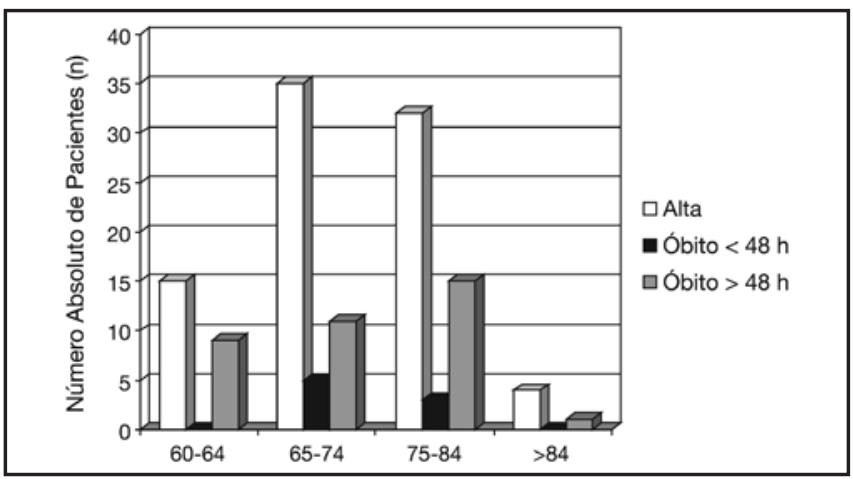

Figura 4 - Relação entre Faixa Etária e Óbito na UTI.

$p>0,05$ (não significativo)

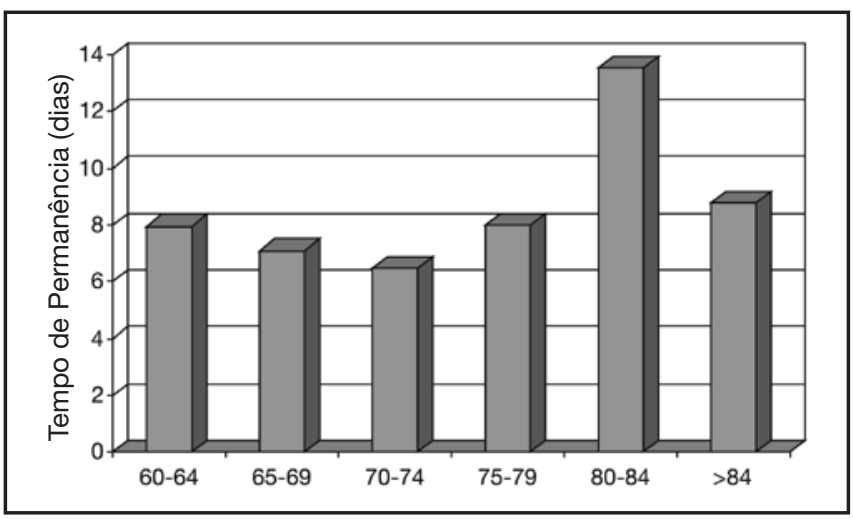

Figura 5 - Relação entre Faixa Etária e Permanência na UTI. $p>0,05$ (não significativo)

Registrou-se mortalidade geral de $33,8 \%$, sendo $27,7 \%$ entre os pacientes com mais de 48 horas de internação na unidade (Tabela 3), com destaque para maior óbito entre os idosos sépticos (61,3\%) (Figura 6). Ressalte-se que a mortalidade prevista, derivada do escore APACHE II, foi superior à mortalidade geral na UTI, resultando disso uma mortalidade padronizada de 0,988.

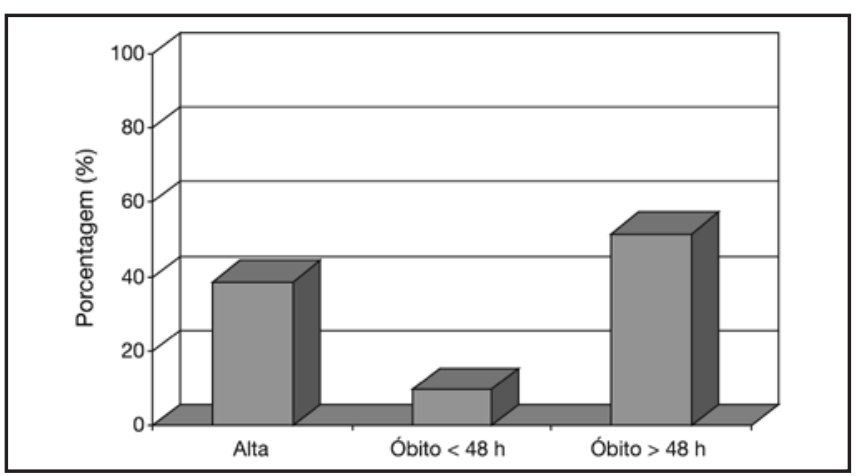

Figura 6 - Desfecho dos Pacientes com Sepse. $p<0,05$

\section{DISCUSSÃO}

Os cuidados de saúde destinados a pacientes idosos têm sido assunto freqüente na literatura durante os últimos anos. O crescimento do número de pessoas com 60 anos ou mais, bem como da expectativa de vida, traduz um aumento nas admissões de pacientes nessa faixa etária nas UTI. Com isso, as comorbidades, a função cognitiva e o estado funcional passam a ser componentes importantes dos cuidados ${ }^{6}$. Dado o alto custo desses cuidados intensivos agudos e o número crescente de pacientes idosos graves necessitando de UTI, é preciso avaliar os fatores relacionados à sua evolução.

No presente trabalho, identificou-se um predomínio do sexo feminino, que ficou ainda mais evidente nos indivíduos muito idosos (85 anos ou mais). Este fato já havia sido notificado em trabalhos anteriores ${ }^{6-9}$. Como demonstrado por Boumendil e col. ${ }^{6}$ e Djaiani e col. ${ }^{7}$, o sexo não influenciou o prognóstico dos pacientes idosos admitidos na UTI.

Reconhece-se que a gravidade da doença aguda influencia a evolução dos pacientes, constituindo a base dos sistemas de escores que são usados regularmente para identificar os pacientes graves ${ }^{7}$. Diferentemente de outros modelos que prevêem a mortalidade, como o SAPS II ${ }^{10}$ e o MPM II ${ }^{11}$, o APACHE II inclui as comorbidades em sua avaliação, mostrando-se um bom método para predizer a evolução dos pacientes idosos. Neste estudo, a média de APACHE II foi $18,2 \pm 7,2$, com mortalidade prevista de $34,1 \%$.

Dentre os sistemas orgânicos acometidos de forma aguda, predominaram as disfunções cardiovasculares e respiratórias. As primeiras estiveram associadas a menores valores de APACHE II, tempo de permanência e mortalidade na UTI. Da mesma forma como demonstrado por Kass e col. ${ }^{12}$, o distúrbio agudo responsável pela admissão à UTI teve relação direta com a sobrevida. A incidência de doenças infecciosas aumenta com a idade, sendo responsável por um terço do óbito em pacientes acima de 65 anos. A sepse é uma condição comum nessa população, com taxa de mortalidade de $20 \%$ a $40 \%$. A deficiência imunológica relacionada à idade e as outras comorbidades favorecem a ocorrência das infecções ${ }^{13,14}$. No presente estudo, a sepse acometeu quase um quarto dos pacientes, com mortalidade geral de $61,3 \%$.

A razão de mortalidade padronizada é uma das medidas comumente usadas para caracterizar a qualidade da UTI. Neste trabalho, o valor foi 0,988. Em países de- 
senvolvidos, documentaram-se valores menores que 1,0; enquanto, no Brasil, Bastos e col. ${ }^{15}$ registraram um valor médio de 1,67. Ressalte-se, porém, que esses autores tiveram, em sua casuística, pacientes de todas as faixas etárias.

Foi demonstrado previamente ${ }^{9,16}$ que os pacientes idosos graves são submetidos a menos procedimentos invasivos e menos intervenções onerosas quando comparados a pacientes jovens com disfunções semelhantes. Essa distribuição preferencial dos serviços hospitalares para pacientes mais jovens não parece ter como base as características dos distúrbios agudos. Talvez, o real motivo seja o fato de alguns médicos ainda acreditarem que os pacientes idosos respondam menos a certas medidas terapêuticas. Porém, estudos anteriores $6,7,9$ mostraram que alguns pacientes idosos se recuperam muito bem após os cuidados intensivos; e que a idade é um critério impróprio para direcionar tais recursos hospitalares, devendo as decisões terapêuticas ser baseadas na capacidade do paciente de se beneficiar do tratamento. No presente trabalho, não houve associação significativa da idade com a mortalidade, nem com o tempo de permanência na UTI. Como já descritos ${ }^{3,8,18}$, não é a idade per se que determina um pior prognóstico; mas sim os fatores associados, tais como a gravidade da disfunção aguda, as comorbidades e o estado funcional antes da admissão na UTI.

Neste estudo, podem-se identificar algumas limitações. Primeiro, o trabalhou focalizou as disfunções agudas, não avaliando o impacto do estado funcional antes da hospitalização. de Rooij e col. ${ }^{17}$ demonstraram que esse fator, incluindo os funcionamentos físico, cognitivo e social, tem se mostrado um importante preditor da evolução hospitalar dos pacientes idosos. Segundo, a mortalidade dos pacientes com idade entre 60 e 84 anos não foi significativamente diferente daquela observada nos pacientes com 85 anos ou mais. Boumendil e col. ${ }^{6}$ e Djaiani e col. ${ }^{7}$ mostraram uma relação direta entre idade maior que 85 anos e pior prognóstico. Talvez essa diferença seja decorrente do pequeno número de pacientes nessa faixa etária neste trabalho. Existem outras limitações inerentes ao próprio sistema APACHE II. Este escore não leva em consideração o estado funcional do paciente antes da admissão à UTI; além de não valorizar complicadores prognósticos freqüentes em pacientes idosos, como desnutrição e delírio. Alguns investigadores ${ }^{17-21}$ já haviam mostrado que essa deficiência do método pode subestimar o prognóstico do idoso da UTI.
Em conclusão, o declínio súbito de qualquer órgão ou função é quase sempre devido à doença aguda, e não decorrente da idade. Portanto, os sintomas na população idosa não devem ser automaticamente atribuídos à velhice, sendo importante atentar para as suas causas potencialmente reversíveis.

\section{REFERÊNCIAS}

01. IBGE / Diretoria de Pesquisas. Coordenação de População e Indicadores Sociais. Gerência de Estudos e Análises da Dinâmica Demográfica. Projeção da População do Brasil por Sexo e Idade para o Período 19802050, Revisão 2004.

02. Nagappan R, Parkin G - Geriatric critical care. Crit Care Clin, 2003;19:253270.

03. Adelman RD, Berger JT, Macina LO - Critical care for the geriatric patient. Clin Geriatr Med, 1994;10:19-30.

04. Knaus WA, Draper EA, Wagner DP et al - APACHE II: A severity of disease classification system. Crit Care Med, 1985;13:818-829.

05. American College of Chest Physicians/Society of Critical Care Medicine Consensus Conference: definitions for sepsis and organ failure and guidelines for the use of innovative therapies in sepsis. Crit Care Med, 1992;20:864-874.

06. Boumendil A, Maury E, Reinhard I et al - Prognosis of patients aged 80 years and over admitted in medical intensive care unit. Intensive Care Med, 2004;30:647-654.

07. Djaiani G, Ridley S - Outcome of intensive care in the elderly. Anaesthesia, 1997;52:1130-1136.

08. Vosylius S, Sipylaite J, Ivaskevicius J - Determinants of outcome in elderly patients admitted to the intensive care unit. Age Ageing, 2005;34:157162.

09. Hamel MB, Teno JM, Goldman L et al - Patient age and decisions to withhold life-sustaining treatments from seriously ill, hospitalized adults. Ann Intern Med, 1999;130:116-125.

10. Le Gall JR, Loirat P, Alperovitch A et al - A simplified acute physiology score for ICU patients. Crit Care Med, 1984;12:975-977.

11. Lemeshow S, Teres D, Avrunin JS et al - Refining intensive care unit outcome prediction by using changing probabilities of mortality. Crit Care Med, 1988;16:470-477.

12. Kass JE, Castriotta RJ, Malakoff F - Intensive care unit outcome in the very elderly. Crit Care Med, 1992;20:1666-1671.

13. Merrien D - Characteristics of infectious diseases in the elderly. Presse Med, 2002;31:1517-1520.

14. Leibovici $L$ - Bacteraemia in the very old. Features and treatment. Drugs Aging, 1995;6:456-464.

15. Bastos PG, Sun X, Wagner DP et al - Application of the APACHE III prognostic system in Brazilian intensive care units: a prospective multicenter study. Intensive Care Med, 1996;22:564-570.

16. Hamel MB, Phillips RS, Teno JM et al - Seriously ill hospitalized adults: do we spend less on older patients? SUPPORT Investigators. Study to Understand Prognosis and Preference for Outcomes and Risks of Treatment. J Am Geriatr Soc, 1996;44:1043-1048.

17. de Rooij SE, Abu-Hanna A, Levi M et al - Factors that predict outcome of intensive care treatment in very elderly patients: a review. Crit Care, 2005;9:R307-R314.

18. Bo M, Massaia M, Raspo S et al - Predictive factors of in-hospital mortality in older patients admitted to a medical intensive care unit. J Am Geriatr Soc, 2003;51:529-533.

19. Ely EW - Optimizing outcomes for older patients treated in the intensive care unit. Intensive Care Med, 2003;29:2112-2115.

20. Ely EW, Stephens RK, Jackson JC et al - Current opinions regarding the importance, diagnosis, and management of delirium in the intensive care unit: a survey of 912 healthcare professionals. Crit Care Med, 2004;32:106-112.

21. Ely EW, Gautam S, Margolin R et al - The impact of delirium in the intensive care unit on hospital length of stay. Intensive Care Med, 2001;27:1892-1900. 